\title{
Metodología para el Estudio de Soldabilidad en Uniones Soldadas
}

\author{
Enrique E. Niebles ${ }^{(1,2)}$, Jimy Unfried ${ }^{(1)}$ y Jaime E. Torres ${ }^{(2)}$ \\ (1) Grupo de Investigación en Materiales, Procesos y Tecnologías de Fabricación - IMTEF, Programa de \\ Ingeniería Mecánica. Universidad Autónoma del Caribe. Calle 90 N. ${ }^{\circ 46-112 . ~ B a r r a n q u i l l a, ~ C o l o m b i a . ~}$ \\ (e-mail: eniebles@uac.edu.co, jimy.unfried@uac.edu.co) \\ (2) Grupo de Investigación en Materiales, Procesos y Diseño - GIMYP, Programa de Doctorado en \\ Ingeniería Mecánica. Universidad del Norte. Km.5 - vía Puerto Colombia. Barranquilla, Colombia. \\ (e-mail: jtorres@uninorte.edu.co)
}

Recibido May. 29, 2013; Aceptado Jul. 17, 2013; Versión final recibida Sep. 11, 2013

\begin{abstract}
Resumen
Se presenta una nueva metodología que describe aspectos relevantes en la caracterización, análisis microestructural y mecánico de aleaciones de aluminio extensible a otro tipo de metales. La metodología está basada en el concepto de soldabilidad y en las técnicas de análisis cualitativas y cuantitativas fundamentadas en el estudio de la literatura, la experimentación, modelación, simulación y validación de resultados. La metodología propuesta contribuye a garantizar el aseguramiento de la calidad de la soldadura, la selección adecuada de ventanas del proceso, condiciones de trabajo y estudios profundos de soldabilidad en construcciones soldadas. Este trabajo genera un documento de apoyo a procesos investigativos y formativos en soldadura, de referencia para profesionales del área de la ciencia de materiales y procesos.
\end{abstract}

Palabras clave: soldabilidad, caracterización microestructural, modelación y simulación, aleaciones de aluminio

\section{Methodology for Weldability Study of Welded Joints}

\begin{abstract}
A new methodology that describes relevant aspects of microstructure characterization and mechanical properties analysis of aluminum alloys, which may be used in other metals, is presented here. The methodology is based on qualitative and quantitative techniques of analysis, and it is substantiated on the weldability concept, which is constructed by means of literature information, experimental methods, modeling, simulation and validation of the results. The proposed methodology helps ensuring the quality of welding, selection of process parameters windows, working conditions and in-depth studies of weldability on welded constructions. This work generates a useful document to support research and learning processes of welding, which could be used as reference for professionals of welding engineering and materials science.
\end{abstract}

Keywords: weldability, microstructure characterization, modeling and simulation, aluminum alloys 


\section{INTRODUCCIÓN}

Un problema muy presente en las industria de construcciones soldadas y en especial las de fabricación naval en aluminio está determinado por la disminución de ciertos valores de indicadores resistentes en las uniones soldadas como son: el límite a la fluencia, la resistencia última a tensión, la elongación, la resistencia a la fatiga en presencia de discontinuidades tales como dislocaciones, poros, fisuras e inclusiones sólidas. Lo anterior es asumido por efecto del calor suministrado y absorbido por la aleación en la zona adyacente al depósito de soldadura; por los procesos de transferencia térmica y cambios de fase respectivos, fenómenos de recristalización presentes y esfuerzos residuales remanentes (Bloem et al., 2007; Pfeifer y Gawrysiuk, 2006; Zander y Sandström, 2009; Torres, 2002; Rossini et al., 2012); esto es, tomando en cuenta que las uniones soldadas son afectadas por las cargas y esfuerzos transmitidos a las juntas, así como por las condiciones de trabajo, geometría y complejidad del producto y/o construcción soldada, generando concentradores de esfuerzos, fatiga, agrietamientos y fracturas (Pfeifer y Gawrysiuk, 2006; Skillingberg y Green, 2007; Asociación Colombiana de Ingeniería Sísmica, 1998).

Como se afirma en la literatura científica sobre este tema, el aporte térmico está asociado a parámetros de soldadura como tensión, intensidad de corriente, velocidad de avance, tipo y flujo de gases de protección, transferencia y altura del arco, posición de soldadura. De manera que el aporte térmico afecta las propiedades físicas y químicas, dado que se generan cambios microestructurales según sea la sensibilidad al calor del material soldado. Estos cambios microestructurales están asociados a la formación de dislocaciones en materiales deformados, a fenómenos de recristalización y tamaño de grano, a la calidad y nivel de precipitados presentes tanto en el interior de los granos como en los límites de grano, a la capacidad de dilución de algunos elementos presentes en las aleaciones, definiendo las transformaciones de fase respectivas, $y$ los esfuerzos residuales remanentes en la unión. (Kuk et al., 2004; Fu et al., 2006; Fortain y Lefebre, 2003). Por tanto, son necesarios estudios de soldabilidad que garanticen la confiabilidad de la unión soldada brindando información valiosa a diseñadores y fabricantes de productos soldados en aluminio. Este artículo tiene por objetivo mostrar la plataforma metodológica utilizada en el desarrollo de la tesis doctoral en ingeniería mecánica "influencia del aporte térmico en uniones soldadas en aluminio AA5083-H116 con proceso GMAW-P”.

\section{SOLDABILIDAD}

La soldabilidad de un material, se define como una propiedad tecnológica que mide la capacidad del material de dejarse unir en forma técnica y económica con los procesos y técnicas de soldadura mediante procedimiento adecuados que aseguren la calidad del depósito de soldadura acorde con las especificaciones establecidas en el diseño. El grado de soldabilidad está determinado en gran manera por la sanidad del material y facilidad a soldarse sin necesidad de recurrir a sendos procedimientos previos, durante y post soldadura. (Maury et al., 2009)

Los diversos enfoques y características consideradas en estudios de soldabilidad son mostrados en la figura 1; El estudio de cada una de las características descritas en la tabla 1 para la soldabilidad de aleaciones de aluminio (Zander y Sandström, 2009; Liu et al., 2006; AWS, 2000; Fukuda, 2012), permiten definir como se dan los fenómenos de transformación microestructural que explican el comportamiento mecánico de la unión soldada en las regiones de soldadura, como deben ser las condiciones de servicio y operativas de la unión afín con la fabricación de componentes soldados, procesos y procedimiento de soldadura utilizados. Además definen la técnica utilizada, aporte térmico y velocidad de enfriamiento en la unión soldada.

En el depósito de soldadura se encuentran productos de la solidificación: estructuras dendríticas y columnares, crecimiento epitaxial, impurezas y frecuentemente discontinuidades y defectos de soldadura. En la zona afectada térmicamente (ZAT) se producen cambios microestructurales producto de fenómenos de recuperación, recristalización y crecimiento de grano. Se conoce además que la soldabilidad en uniones soldadas está determinada entre otros por los siguientes factores: i) Compatibilidad química y fluidez del material base y de aporte; ii) Temperatura de fusión, calor especifico, alta conductividad y nivel de expansión térmica; iii) Estabilidad del arco, naturaleza refractaria del óxido de aluminio, características de color similares cerca del punto de fusión y atmosfera de protección; iv) Requerimientos de precalentamientos y tratamientos térmicos antes, durante y post soldadura; v) Tensión superficial característica del depósito de soldadura fundido; vi) Consideraciones de esfuerzos residuales, rigidez, ductilidad, fatiga y resistencia a la corrosión tanto del material base, material de aporte y depósito de soldadura; y vii) Consideraciones de diseño y desarrollo para la fabricación y ensamble de productos soldados. 


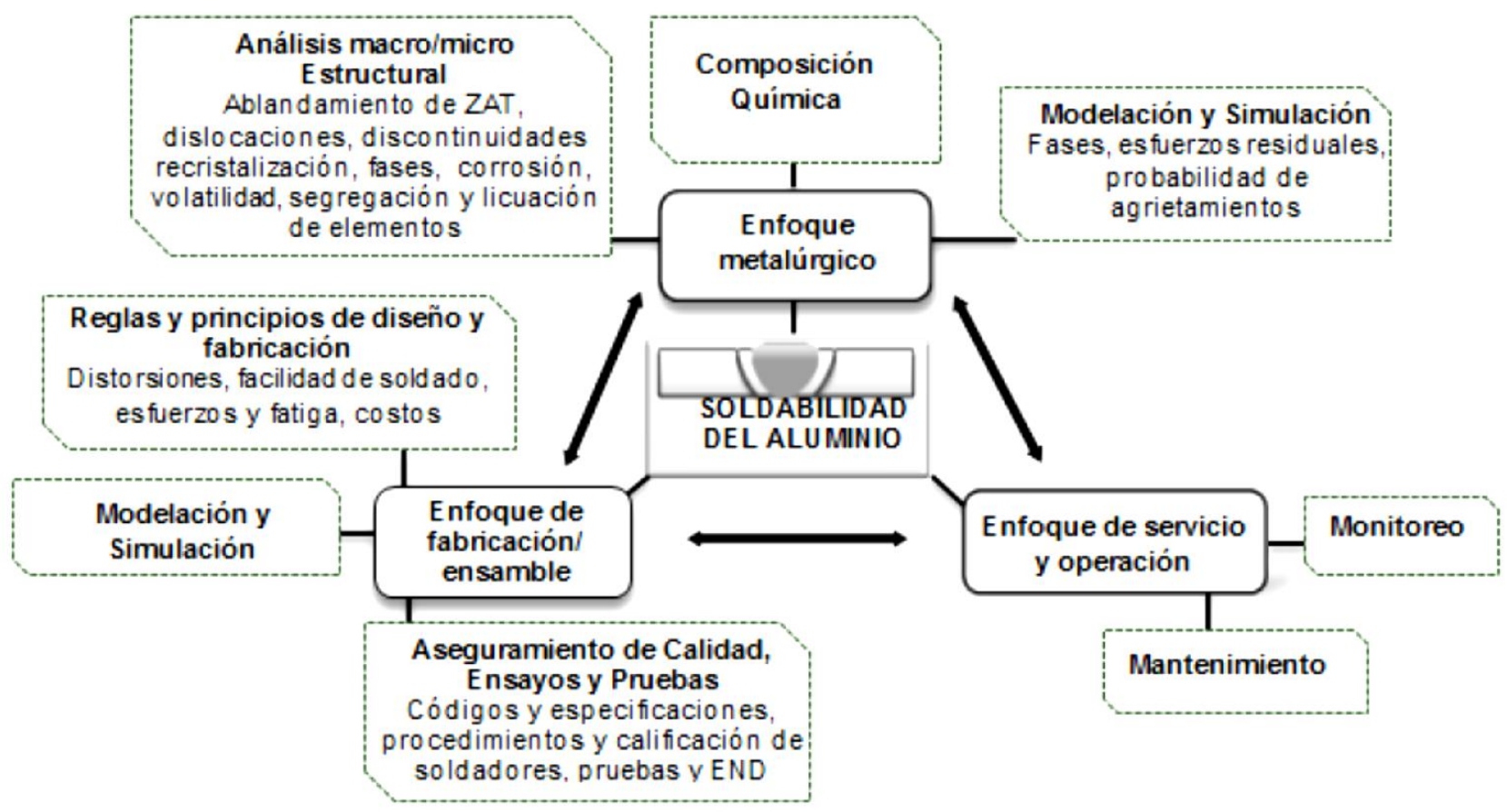

Fig. 1: Enfoque y aspectos relevantes en el estudio de la soldabilidad.

Un control efectivo del calor aportado se logra, además, utilizando procesos adecuados de soldadura, con gas de protección GMAW, GTAW y por fricción-agitación (Yazdipour et al., 2011) y otras técnicas de soldadura que garanticen una ZAT mínima.

\section{METODOLOGÍA PARA LA INVESTIGACIÓN EN SOLDADURA}

La metodología para el desarrollo de proyectos de investigación requiere de claridad, pertinencia, coherencia, validez y confiabilidad en los métodos y técnicas cuantitativas y cualitativas utilizadas en relación con el cumplimiento de los objetivos y los resultados esperados, en un marco de tiempo ajustado a un presupuesto y cronograma de actividades. En la figura 2 se muestran las fases y actividades más importantes que han aplicado los autores en el desarrollo de proyectos de investigación.

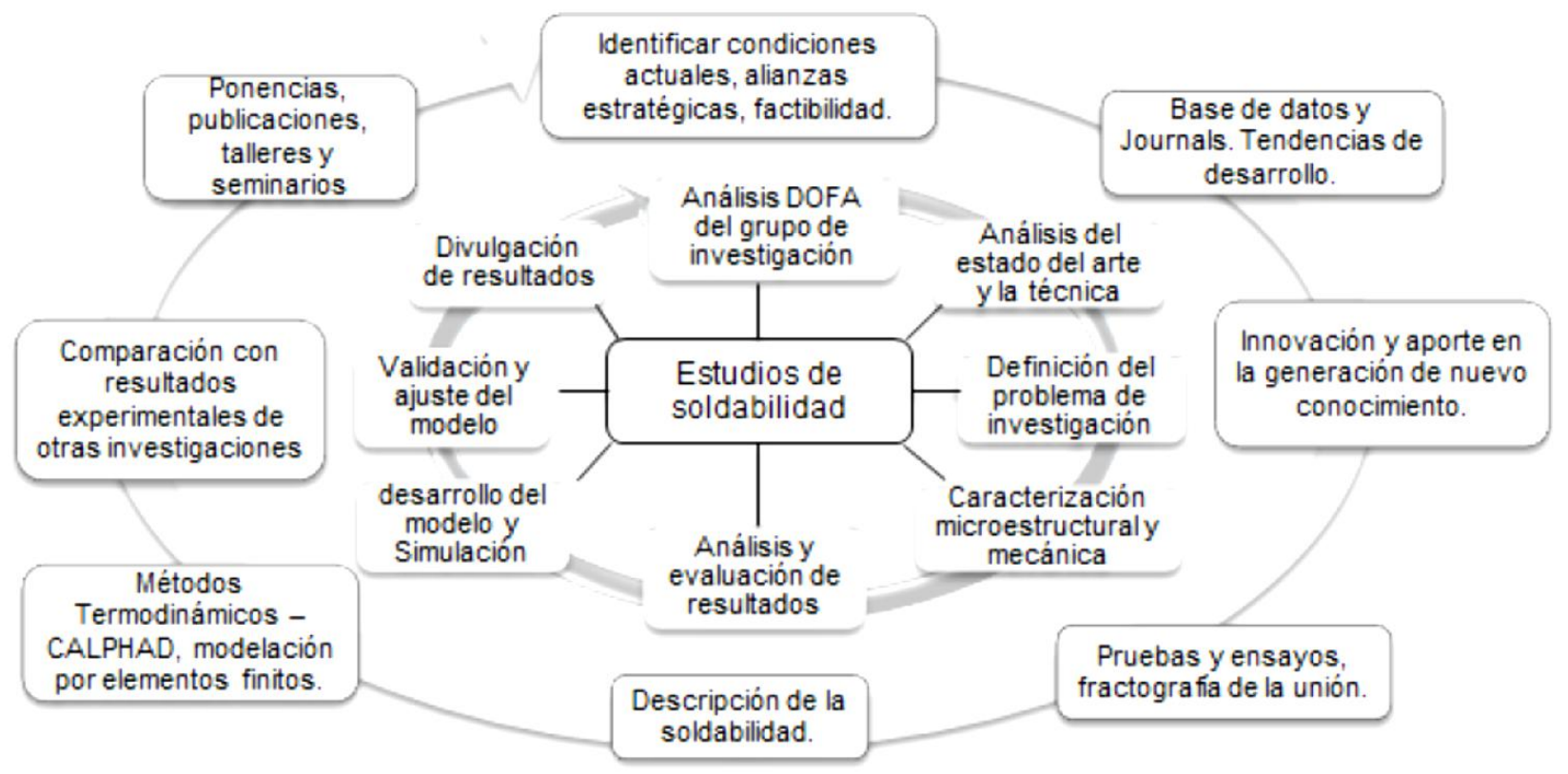

Fig. 2: Metodología para estudios de soldabilidad 
La formulación de estrategias en la tabla 2, es presentada en coherencia con los resultados obtenidos de la valoración ponderada de los factores DOFA apuntando a aprovechar las oportunidades y fortalezas que dan viabilidad al proyecto, de tal manera que se minimicen los efectos de riesgo en el proyecto.

II. Análisis del estado del arte. Se revisará que la información registrada en journals y revistas especializadas y seriadas, catálogos de fabricantes, blogs de expertos, etc., esté actualizada, sea coherente y pertinente con el tema de estudio permitiendo desarrollar el estado del arte y la técnica así como el marco teórico referencial en función de caracterizaciones y análisis microestructurales y mecánicos realizados en el material de estudio y afines, modelos obtenidos de transformaciones de fase y cinética de precipitados presentes en la unión, metodología y análisis de soldabilidad de las aleaciones, tendencias de desarrollo, procesos de soldadura, variables e influencia en la unión soldada y todo aquello que en la búsqueda aporte al objeto de investigación, desarrollo e innovación.

III. Definición del problema, justificación y variables de análisis. Con base en la información obtenida y registrada, producto de la fase II, que permite definir la factibilidad y ventanas de la investigación se procede a definir la situación problema y pregunta de investigación ¿Cuál es la influencia específica del aporte térmico en la soldabilidad de la aleación de aluminio AA5083-H116 en términos de la macro y microestructura y las propiedades mecánicas de la aleación? Desde luego que en esta pregunta es necesario tener en cuenta la sensibilidad a la disminución de los indicadores resistentes, la probabilidad de formación de fisuras, poros y tensiones residuales para poder establecer correlaciones con los índices de desempeño de la junta soldada bajo cargas dinámicas y cuasi estáticas, lo que permitirá asegurar la integridad de la unión soldada.

A continuación se identifican variables de análisis y especificaciones correspondientes con el tema de investigación, mediante la elaboración de un análisis funcional (Aguayo y Soltero, 2003; Ulrich y Eppinger, 2004; Otto y Wood, 2001) del objeto de investigación que describa cuales son los elementos funcionales a tener en cuenta para el desarrollo de la investigación. Se establecen las especificaciones iniciales, mostradas en la tabla 3; se justifica la propuesta de investigación, en términos de la innovación y aporte científico y tecnológico a la generación de nuevo conocimiento en los ámbitos local, nacional e internacional, así como del impacto y beneficios obtenidos productos del fortalecimiento del sector productivo y de los grupos de investigación.

Tabla 3. Lista de especificaciones: $\mathbf{C}=$ cliente, $\mathbf{I}$ =investigador, $\mathbf{R}=$ requerimiento, $\mathbf{D}=$ deseado.

\begin{tabular}{|c|c|c|c|}
\hline \multicolumn{2}{|l|}{$\begin{array}{c}\text { Uninorte } \\
\text { Empresa Cliente: }\end{array}$} & \multicolumn{2}{|c|}{$\begin{array}{l}\text { Fecha de inicio: Marzo de } 2011 \\
\text { Investigadores: Enrique E. Niebles Nuñez, Jaime Torres S. } \\
\text { Jimy Unfried S. }\end{array}$} \\
\hline \multicolumn{4}{|c|}{ Proyecto de Tesis Investigación: Influencia del aporte térmico en la soldabilidad de la aleación AA5083-H116 } \\
\hline \multicolumn{4}{|c|}{ Lista de especificaciones iniciales } \\
\hline Concepto & Propone & Tipo R/D & Determinaciones \\
\hline Parámetros del proceso & C & $\mathrm{R}$ & \\
\hline Diseño de junta & $C+1$ & $\mathrm{R}$ & \\
\hline Composición química de metal base & 1 & $\mathrm{R}$ & \\
\hline Ensayos/ pruebas mecánicas & $\mathrm{C}+\mathrm{I}$ & $\mathrm{R}$ & \\
\hline Observación microestructural & 1 & $\mathrm{R}$ & \\
\hline Aspectos normativos & $C+1$ & D & \\
\hline
\end{tabular}

IV. Caracterización microestructural y mecánica de materiales base, de aporte y depósito de soldadura.

En esta fase se preparan los especímenes de prueba, se ejecutan soldaduras y realizan ensayos y pruebas mostrados en la figura 3, requeridas para la caracterización del metal de base, del depósito de soldadura y de la unión soldada así como la determinación del comportamiento mecánico en términos de la resistencia última y a fluencia, ductilidad de la unión y estudio microestructural profundo en las regiones de soldadura especialmente en la ZAT (Scott et al., 2006; Smallman y Bishop, 1999; Kou, 2003; Totten y MacKenzie, 2003). Es recomendable además complementar el estudio mediante análisis fractográfico de las probetas de tensión, tanto del metal base como de las soldaduras, para determinar el comportamiento a la fractura de la unión soldada y correlacionar los resultados con los indicadores de resistencia mecánica. Las actividades necesarias para el desarrollo de esta fase se describen a continuación. 


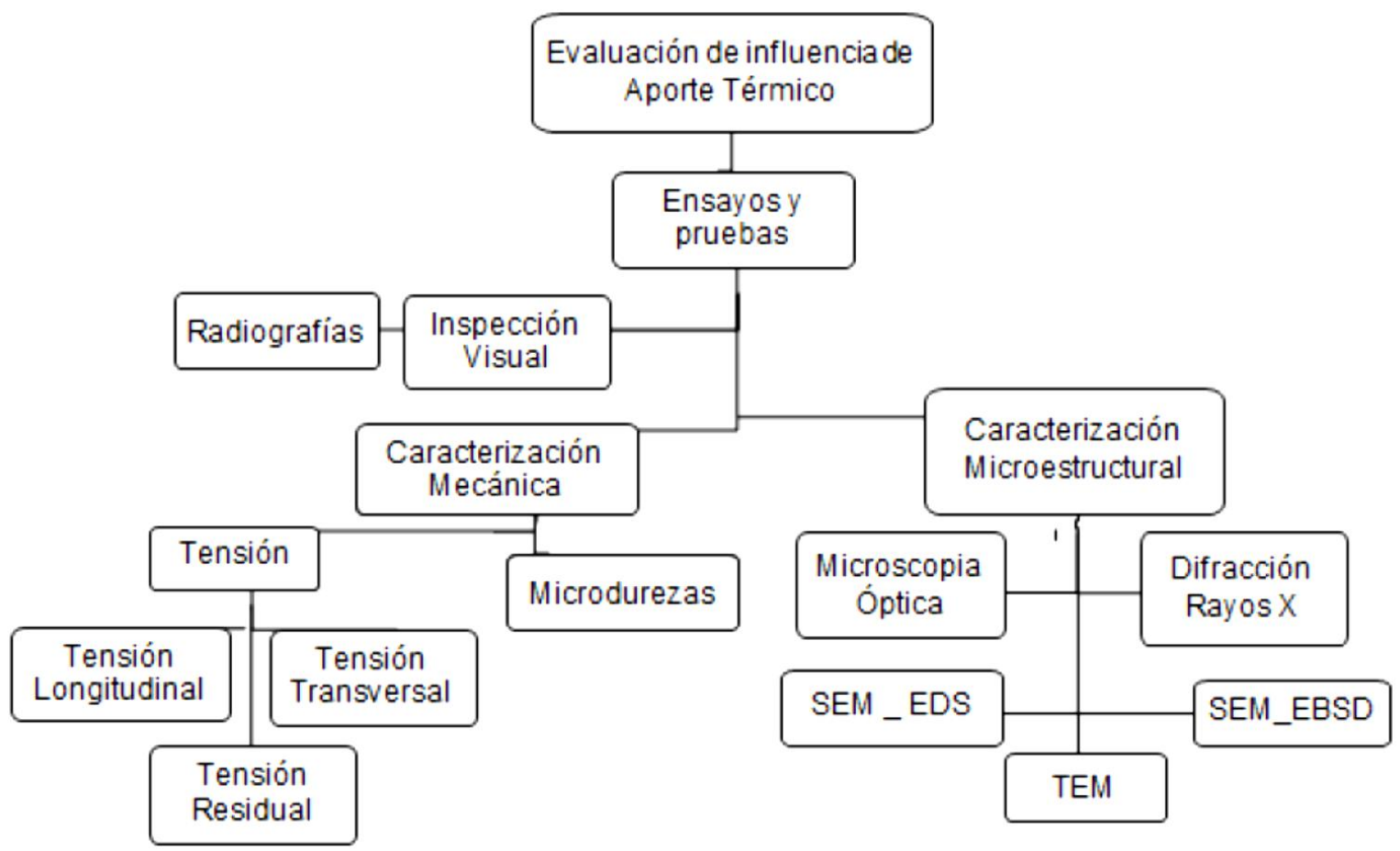

Fig. 3: Pruebas y ensayos aplicados para la caracterización microestructural y mecánica de las uniones soldadas.

Elaboración del diseño experimental. Este actividad se lleva a cabo utilizando herramientas informáticas de apoyo, donde es común utilizar diseños factoriales completamente aleatorizados de un solo factor fijo (aporte térmico) y diseño de bloques completos aleatorizados de un solo factor fijo siempre definiendo las variables de respuesta y el número de observaciones por réplica de las variable en estudio. El tamaño de muestra para la fase definitiva se obtendrá del desarrollo de soldaduras previas y mediciones realizadas para la estimación de la varianza, con esto se disminuye el estudio en la correlación de variables y el número de ensayos a realizarse en el experimento (Benyounis y Olabi, 2008; Montgomery, 2008; Kuehl, 2001). Se espera obtener pruebas de hipótesis acerca de la igualdad e interacción de efectos utilizando análisis de varianza - ANOVA del experimento, y verificando por análisis de residuales el supuesto de normalidad, homogeneidad de varianzas, autocorrelación de residuales vs tiempo y por último comparaciones múltiples si existe diferencias entre las medias de los tratamientos.

Puesta a punto de equipos y máquinas. Esta es una actividad transversal y se desarrolla durante todo el proyecto, cuando se requiera. La revisión, calibración y puesta a punto de equipos de soldadura, máquinas de ensayos, y demás equipos de laboratorios son necesarias para garantizar la confiabilidad y repetitibilidad de los procedimientos y resultados esperados.

Caracterización del comportamiento mecánico y microestructural del metal base. Esta fase debe permitir la construcción de la argumentación para la hipótesis del comportamiento mecánico de la aleación y la evolución microestructural y química de la misma antes de la soldadura. Se realizan: análisis de la composición química del material base, ensayos de tensión transversal y barrido de microdurezas, análisis de microscopia óptica.

Entrenamiento y calificación de soldadores según proceso de soldadura, material y tipo de unión. Para el desarrollo de las soldaduras es indispensable entrenar y evaluar el desempeño de los soldadores que intervendrán en el proyecto con el fin de minimizar y bloquear los efectos del soldador en la fase experimental de ejecuciones de soldaduras. Para el caso de soldadura de aluminio en componentes estructurales se sigue las indicaciones del código AWS D1.2 - Structural Welding Code- Aluminum (AWS, 2003). El entrenamiento y posterior calificación del soldador (Niebles y Arnedo, 2009) es realizado con un material de características iguales, o similares, al que se le va a realizar el estudio de soldabilidad, según disponibilidad y costos.

Desarrollo de ensayos previos de soldaduras. En paralelo con la actividad de caracterización del metal base se recomienda una fase pre-experimental que permita identificar los valores máximos y mínimos de parámetros de soldadura como voltaje, intensidad y velocidad de soldadura que aproximen a los valores 
para las entradas de calor que se utilizaran en la fase experimental definitiva. El diseño de los cupones a soldar, mostrados en la figura 4, depende del alcance y objetivos del proyecto de investigación y debe seguir fielmente lo planificado en términos de los entrenamientos y calificaciones de los soldadores las pruebas, ensayos, inspecciones y réplicas. Para lograr el éxito de esta fase se debe realizar una evaluación del comportamiento mecánico de la unión y los cambios estructurales de nivel macro y micro que permitan diseñar procedimientos de soldadura aplicables para la fase definitiva de la investigación, se recomienda para la toma de decisiones además del diseño de experimentos utilizado, complementar el análisis con métodos de criterios ponderados (Maury et al., 2009; Aguayo y Soltero 2003) donde cada criterio es sometido a evaluación de expertos.
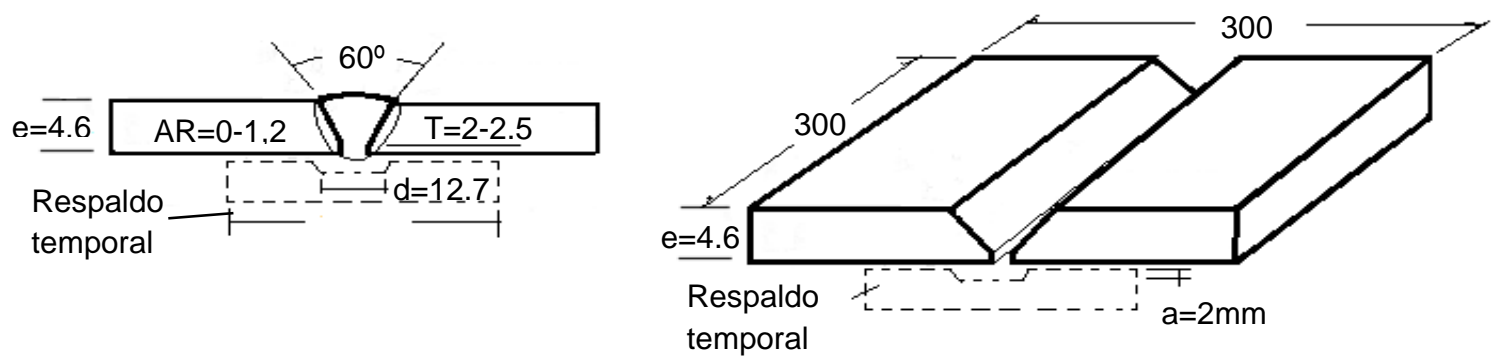

Fig. 4: Diseño de junta utilizado en la fase de soldaduras definitivas. AR=Abertura de raíz, E=espesor, T=Talón. Medidas en milímetros.

Desarrollo definitivo de juntas soldadas. El diseño de los cupones a soldar en esta fase toman en cuenta los resultados obtenidos en la fase previa de soldaduras. Los cortes, las preparaciones de bordes y biseles de los cupones y la ejecución de la soldadura de los mismos deberán ejecutarse acorde con el diseño de experimentos y procedimiento de soldadura especificado.

Determinación de ciclos térmicos. Se obtienen las temperaturas y tasas de enfriamiento de las uniones soldadas en las diferentes regiones de soldadura, útiles para estudios con técnicas de calorimetría diferencial de barrido DSC. Permiten relacionar los cambios microestructurales de la unión soldada con los fenómenos de recristalización y transformaciones de fase presentes. La temperatura suministrada al material se determina con análisis de imágenes termográficas y termocuplas acopladas a un sistema de adquisición datos y análisis Lab-view.

Prueba y ensayos mecánicos a realizar. Se inicia con la inspección visual de los cupones soldados, y puede continuarse con las radiografías analizando la mayor confluencia de discontinuidades posibles. Posterior a las inspecciones iniciales se procede al trazado, corte e identificación alfa numérica de los especímenes para los ensayos mecánicos y las pruebas metalográficas. La ubicación de los especímenes para los ensayos de tensión transversal y longitudinal se muestran en la figura 5a. La preparación del espécimen de tensión transversal se realiza acorde con el código para soldaduras de estructuras de aluminio AWS D1.2. Para la preparación del espécimen de tensión longitudinal se toma como referencia a escala, lo especificado en la norma ASTM B557-10, como se muestra en la figura 5b. Lo que se espera obtener del ensayo de tensión son los valores de resistencia última, a fluencia y ductilidad en correlación con las discontinuidades en la unión.

Caracterización del depósito de soldadura. Con esta actividad se busca sustentar la composición química del material depositado de soldadura en estudio acorde con el material de aporte utilizado y procedimiento normativo recomendado. Se requiere realizar un análisis químico acorde con la norma ASTM E34-11 y diseño de la junta especificado en AWS A5.10/A5.10M: 1999 R (2007)

Procedimiento para la realización de barridos y mapas de microdurezas. La construcción de las curvas de barridos, como se muestra en la figura 6 , y mapas de microdurezas para identificar el comportamiento de la dureza en toda la junta soldada permiten identificar los máximos y mínimos de dureza, homogeneidad y su relación con la posición en cada una de las regiones de soldadura.

Preparación y realización de análisis metalográfico con microscopio óptico. Con los resultados de macroataque y las curvas de barridos de microdurezas se determinan la dimensión y macro morfología de la ZAT, mientras que con el estudio de microscopia óptica (MO) se analizan áreas grandes e identifican partículas de segunda fase estadísticamente representativas, identificando el tamaño y estado del grano (Fu et al., 2006; Oguocha et al., 2008; Ma y den Ouden, 1999). 

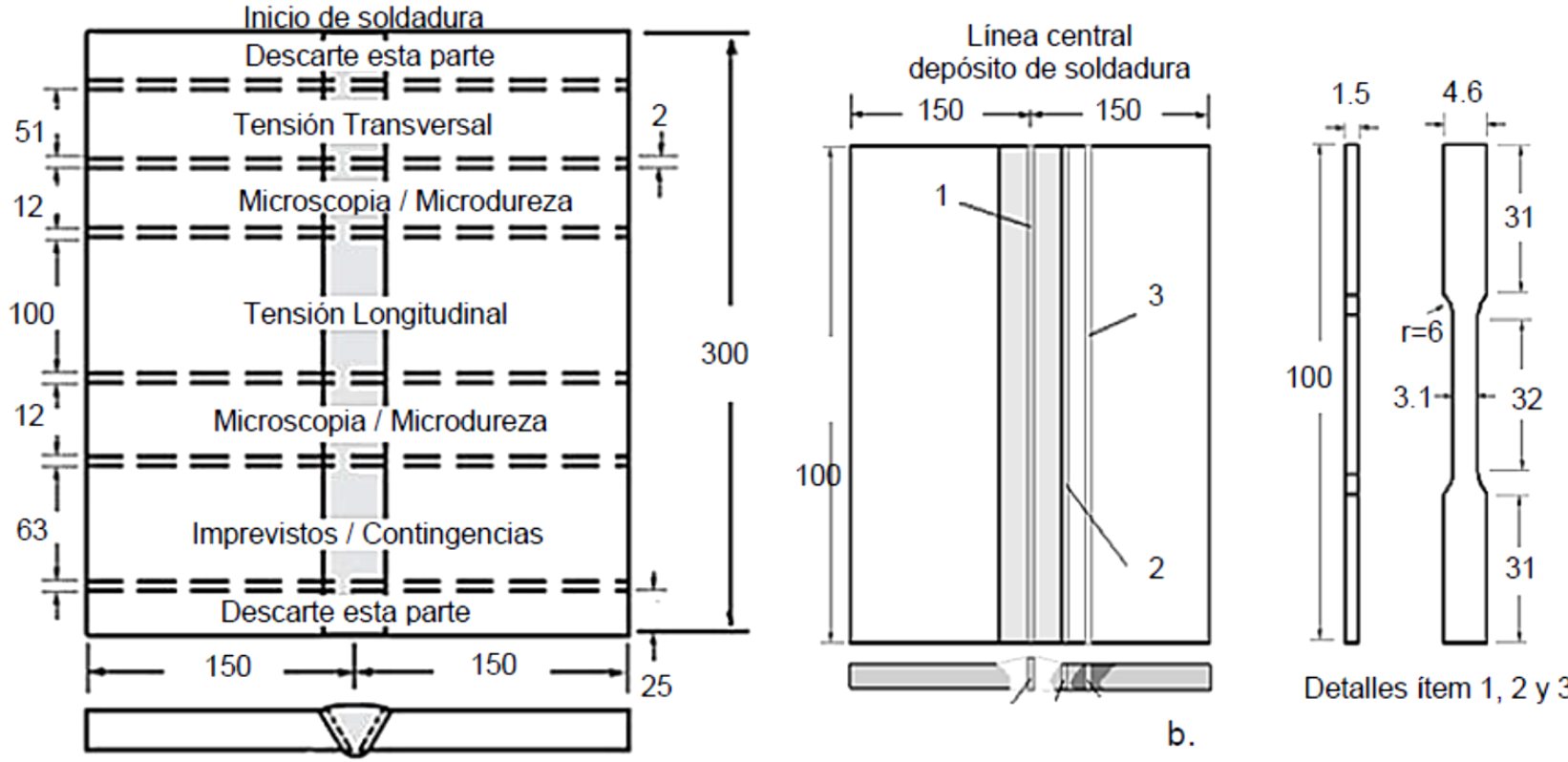

a.

b.

Fig. 5: Especimenes de prueba utilizados fase de soldaduras definitivas. a. Ubicación de especimenes para una replica, b. Espécimen de prueba para ensayo de tensión longitudinal, en regiones $1=Z P F, 2=Z G G, 3=$ ZGF y metal base. Todas las medidas en milimetros

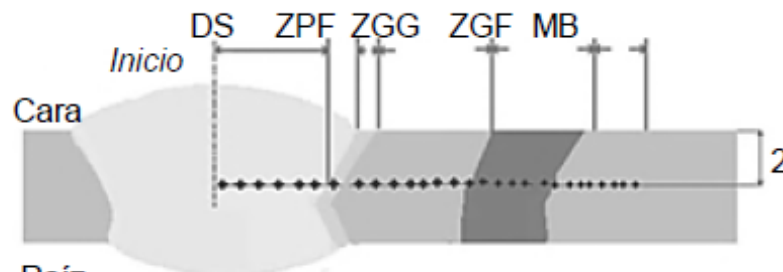

Raíz

a.

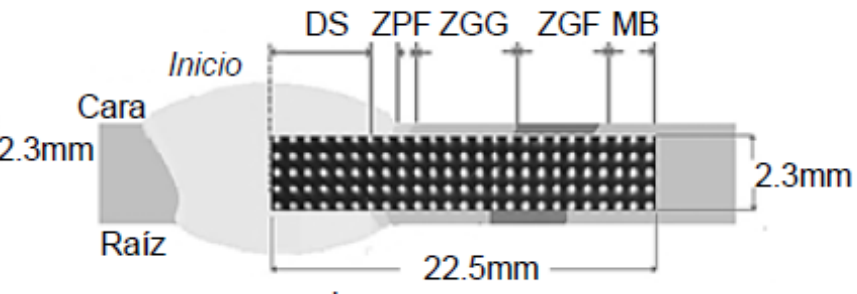

b.

Fig. 6: Esquema del barrido y mapas de microdureza HV para juntas soldadas. Fase de soldaduras definitivas. a. Secuencia de barrido, b. Mapa de durezas. DS=Depósito de soldadura, ZPF=Zona parciamente fundida, $Z G G=Z o n a$ de grano grueso, $Z G F=Z$ ona de grano fino, $M B=$ Metal base.

En esta fase de microscopia óptica no es posible identificar precipitados, ni dislocaciones por lo que su alcance es bastante limitado. (ASM International, 1984; Padilha y Siciliano Jr., 2005). La preparación de las muestras metalográficas (ASTM International, 2011) se realiza para el análisis del metal base y de las uniones soldadas. Con ayuda de un software analizador de imágenes se medirá el tamaño de grano. Tomando como base observaciones preliminares en el microscopio óptico, como se muestra en la figura 7 , se obtienen imágenes en las regiones de soldadura a magnificaciones de 50X, 100X, 200X y 500X en cada región de soldadura identificada.

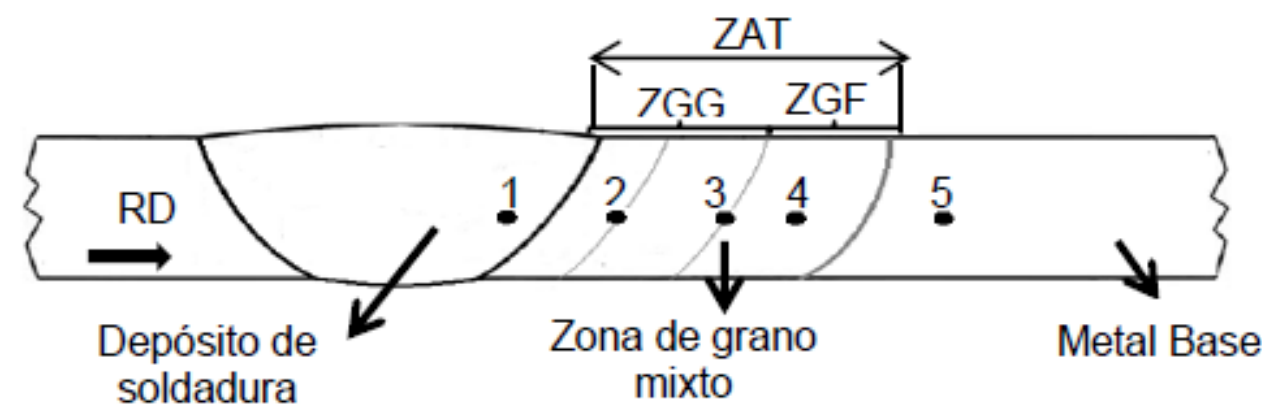

Fig. 7: Ubicación de imágenes en el microscopio óptico. Aleaciones soldadas de aluminio AA5083-H116. RD=Dirección de laminado 
Preparación y realización de análisis microestructural con técnicas de microscopía electrónica de barrido SEM. (ASM International, 1984; Padilha y Siciliano Jr., 2005) Las ventajas del análisis SEM son: Fácil preparación del espécimen, mayor gama de magnificación que la MO. Esta fase permite la obtención de imágenes en los modos Electrón Secundario (SE) y retrodispersado (BSE) para estudiar la estructura de granos y subgranos en las regiones de soldadura, así como la identificación de precipitados y partículas de segunda fase.

Con el complemento de espectrometría dispersiva de Energías - EDS se puede obtener imágenes en los modos SE y BSE, así como microanálisis de composición química por EDS con rayos $\mathrm{x}$, en alta resolución para realizar una caracterización básica completa de los precipitados y la variación y composición química en la interface y regiones de soldadura.

Con el microanálisis se puede detectar perdidas de elementos aleantes e identificar fases en la frontera del grano causantes de fisuración y el desencadenamiento de la corrosión intercristalina. (Katsas et al., 2006) Los parámetros de trabajo utilizados, para el estudio de la soldabilidad en aleaciones soldadas de aluminio AA5083-H116, en un microscopio FEG-SEM al aplicar la técnica SEM modos SE y EDS para el análisis de la microestructura en las diferentes regiones de soldadura de la muestras en estudio son: abertura de lente objetiva $=5(30 \mu \mathrm{m})$, tamaño de spot $=5-6$, distancia de trabajo $=10 \mathrm{~mm}$, aceleración de voltaje $=20 \mathrm{kV}$, magnificación $=1000 \mathrm{X}, 10000 \mathrm{X}$ y $30000 \mathrm{X}$. En el modo EDS el software utilizado para la adquisición de datos fue el software "INCA" en la opción de full acquisition mode con Dead time de $20 \%$ a $30 \%$. Los parámetros utilizados para la adquisición del espectro LiveTime $=30 \mathrm{~s}$, Spectrum Range $=0-20 \mathrm{kV}$ y number of Channels $=2$.

Así mismo es posible utilizar la técnica de difracción de electrones retrodispersados - EBSD para análisis de texturas y cambios microestructurales en la ZAT que permitan medir el nivel de recristalización en las juntas soldadas para luego correlacionar estos datos con la dureza. Para la evaluación microestructural con la técnica de EBSD no es necesario atacar las muestras. La muestra no debe evidenciar rayado alguno al observarla en el MO. Los parámetros de trabajo, para el estudio de la soldabilidad en aleaciones soldadas de aluminio AA5083-H116, en un microscopio FEG-SEM con software de adquisición de datos "HKL Fast Adquisitión" al aplicar la técnica de EBSD para el análisis de la microestructura en las diferentes regiones de soldadura de la muestras en estudio son: Inclinación de la muestra $=70^{\circ}$, abertura de lente objetiva $=3$ $(50 \mu \mathrm{m})$, tamaño de spot $=5$, distancia de trabajo $=23 \mathrm{~mm}$, aceleración de voltaje $=15 \mathrm{kV}$, paso $=0.5 \mu \mathrm{m}$ $0.75 \mu \mathrm{m}$, magnificación $=1000 \mathrm{X}$, indexación promedio de patrones EBSP en regiones de soldadura $=83 \%$. Con el fin de minimizar el ruido en las imágenes obtenidas, producto de la irregular indexación de los patrones de Kikuchi correspondiente con la muestra en estudio, es recomendable el uso de filtros estándar con repetidas iteraciones hasta conseguir reducir el ruido a cero. Para el análisis de desorientaciones con contornos de bajo ángulo (subgranos), y con el fin de minimizar el ruido de orientación en las imágenes obtenidas, (Humphreys, 2001) se utilizaron filtros Kuwahara con dos o más iteraciones.

Preparación y realización de análisis microestructural con técnicas de calorimetría diferencial de barrido DSC. Esta fase permite caracterizar térmicamente las reacciones de precipitación y analizar la energía de activación durante la recuperación o recristalización de la ZAT. (Padilha y Siciliano Jr., 2005; Park y Ardell, 1989) Se realiza sobre el metal base tratado térmicamente a diferentes temperaturas y tiempos de recocido. Los resultados obtenidos deben ser relacionados con los resultados del análisis de microscopia para el estudio de recristalización y transformaciones de fase presentes.

Preparación y realización de análisis de difracción. Esta fase permite la identificación de fases y textura de grano por medio de un difractómetro de rayos $\mathrm{X}$. Se realiza solo si un análisis previo estereográfico de fases (precipitados) indica una fracción volumétrica mayor al 5\%. (Padilha y Siciliano Jr., 2005) Es apropiado tomar en cuenta durante su análisis, que un error residual ponderado Rwp entre 2 y $10 \%$ indica un buen resultado de los difractogramas obtenido. (Guinebretière, 2007) Para estudios en la identificación de fases, es conveniente complementar sus resultados con los resultados obtenidos mediante técnicas de microscopia electrónica en las variantes SEM-EDS y SEM-EBSD.

V Desarrollo del modelo y simulación. En función de datos y cartas de control del material de estudio, ventanas del proceso y desarrollos experimentales, composición química del metal base y del depósito de soldadura, temperaturas pico y tasas de enfriamiento presentes en las zonas de soldadura se pueden desarrollar modelizaciones y simulaciones del comportamiento de las transformaciones de fase y cinética de precipitados presentes en la microestructura durante la soldadura con los cuales es posible estimar la reducción o modificación de las propiedades mecánica y la probabilidad de agrietamientos en caliente en función de los ciclos térmicos sobre la ZAC utilizando para ello un método que permita lograr esto, como el CALPHAD (Computer Coupling of Phase Diagram and Thermochemistry) (Zander y Sandström, 2009; Saunders y Miodownik, 1998; Unfried et al., 2009) Se pueden desarrollar además modelizaciones por 
elementos finitos para definir el comportamiento de las tensiones residuales en función de los parámetros de soldadura, geometría y restricciones de la unión, así como procesos de solidificación del material. (Liu y Adams, 1998; Anca et al., 2011; Lindgren, 2006) Se debe validar y ajustar el modelo comparándolo con los resultados experimentales obtenidos con el fin de generar conclusiones generales e identificar nuevas ventanas de investigación.

VI Análisis y evaluación de resultados. Para identificar que aporte térmico favorece en mejor medida la soldabilidad de la aleación AA5083-H116, se debe hacer un análisis de los resultados obtenidos que defina la soldabilidad de las aleaciones soldadas en aluminio AA5083-H116 tomando como base la información obtenida: i) con la caracterización experimental en el comportamiento microestructural y mecánico del metal base y las regiones de soldadura con especial énfasis en la ZAT, ii) con el modelo y simulación desarrollados, iii) con los resultados del diseño experimental; y por último ponderar los criterios y variables seleccionados para el estudio mediante métodos de criterios ponderados (Aguayo y Soltero, 2003; Niebles y Arnedo, 2009).

VII Productos e impactos esperados. Incluye discriminar los resultados/productos que corresponden a nuevo conocimiento científico o tecnológico, a nuevos desarrollos tecnológicos, al fortalecimiento de la capacidad científica local, nacional e internacional, a la formación y consolidación de redes de investigación y de cooperación científica internacional; así como identificar el impacto social y ambiental sobre la comunidad el entorno que lo rodea, la economía, productividad y competitividad empresarial, que puedan verificarse a través de trabajos de grado o tesis de maestría o doctorado, realización de cursos relacionados con las temáticas de los proyectos, publicaciones científicas, libros, presentación de ponencias en eventos, productos o procesos tecnológicos, patentes, normas, etc.

VIII Divulgar el conocimiento en eventos y publicaciones científicas. Es importante difundir los resultados de la investigación en términos del aporte científico/tecnológico, el impacto generado, y las ventanas de investigación para futuros proyectos o continuidad de la investigación con mayor alcance. Se recomienda publicar en revistas seriadas y de alto impacto en el sector productivo y académico. (Day y Gastel, 2006; Valderrama, 2005)

\section{CONCLUSIONES}

En referencia a lo anteriormente expuesto, se concluye lo siguiente:

i) Desarrollar estudios de soldabilidad implica el conocimiento de amplias y variadas técnicas de caracterización mecánica y microestructural. Más aún implica definir el orden y correlación de estas técnicas, de tal manera que permitan alcanzar los objetivos propuestos en el estudio.

ii) La metodología utilizada en la presente investigación ha permitido estudiar el comportamiento mecánico de las uniones soldadas en aleaciones de aluminio AA5083-O y AA5083- $\mathrm{H} 116$. El análisis de los resultados de los ensayos mecánicos, ha permitido evaluar la respuesta mecánica de la unión en función de los parámetros de soldadura obtenidos y especialmente en función del calor aportado.

iii) La metodología utilizada en la presente investigación ha permitido estudiar la microestructura de la unión y especialmente de la zona afectada térmicamente, evaluando los niveles de recristalización y presencia de segundas fases en la unión soldada.

iv) La fase de simulación numérica permitió identificar las transformaciones de fase en equilibrio de la aleación y definir la secuencia de solidificación del depósito de soldadura y fases presentes, lo que no es evidente con la caracterización microestructural.

v) La metodología se convierte en una herramienta de trabajo que facilita a estudiantes, técnicos e ingenieros y personal afín con la ingeniería de soldadura, con poca o nula experiencia en estudios de caracterización mecánica y microestructural, desarrollar investigaciones en el área de soldabilidad de aleaciones metálicas.

\section{REFERENCIAS}

Aguayo, F., y Soltero, V. M., Metodología del diseño industrial. México D.F.: Alfaomega, RA-MA (2003).

AWS, American Welding Society, Guide for the Gas Shielded Arc Welding of Aluminum and Aluminum Alloy Pipe. American Welding Society - AWS (2000).

AWS, American Welding Society, AWS D1.2/D1.2M:2003 Structural Welding Code - Aluminum. Miami, Florida: American Welding Society. (2003). 
Anca, A., Cardona, A., Risso, J., y Fachionotti, V., Finite element modeling of welding processes. Applied Mathematical Modelling, 688-707 (2011).

ASM International., Aluminum Properties and Physical Metallurgy. (J. Hatch, Ed.) ASM International (1984). Asociación Colombiana de Ingeniería Sismica., Normas Colombianas de diseño y construcción sismo resistente NSR - 98 Tomo 2. Santa Fe de Bogotá (1998).

ASTM International., E3-11 Standard guide for praparation of metallographic specimens. ASTM International (2011).

Benyounis, K., y Olabi, A., Optimization of different welding processes using statistical and numerical approaches - A reference guide. Advances in Engineering Software, 39(6), 483-496 (2008).

Bloem C., S., Amigó, V., y Salvador, M., Comportamiento a Fatiga de Uniones Soldadas GMAW de la Aleación de Aluminio AA 7020. Revista de Metalurgia, 43(2), 111-116 (2007).

Day, R. A., y Gastel, B., How to write and publish a scientific paper. Cambridge New York: Cambridge (2006).

Fortain, J. M., y Lefebre, P., Patente № US 6,586,700 B2. United States (2003).

Fu, G., Tian, F., y Wang, H., Studies on softening of heat-affected zone of pulsed-current GMA welded AlZn-Mg alloy. Journal of Materials Processing Technology, 180(1-3), 216-220 (2006).

Fukuda, T., Weldability of 7000 series aluminium alloy materials. Welding International, 26(4), 256-269 (2012).

Guinebretière, R., X-ray Diffraction by Polycrystalline Materials. Newport Beach: ISTE Ltd (2007).

Humphreys, F., Review: Grain and subgrain characterisation by electron backscatter diffraction. Journal Materials Science(36), $3833-3854$ (2001).

Katsas, S., Nikolaou, J., y Papadimitriou, G., Microstructural changes accompanying repair welding in 5xxx aluminium alloys and their effect on the mechanical properties. Materials \& Design, 27(10), 968-975 (2006).

Kou, S., Welding Metallurgy. New Jersey: Wiley Interscience (2003).

Kuehl, R., Diseño de experimentos. México: Thomson (2001).

Kuk, J., Jang, K., Lee, D., y Kim, I., Effects of temperature and shielding gas mixture on fatigue life of 5083 aluminum alloy. Journal of Materials Processing Technology, 155-156, 1408-1414 (2004).

Lindgren, L., Numerical modelling of welding," Computer methods in applied mechanics and engineering. Computer Methods in Applied Mechanics Engineering, 6710-6736 (2006).

Liu, R.-P., Dong, Z.-J., y Pan, Y.-M., Solidification crack susceptibility of aluminum alloy weld metals. Transactions of Nonferrous Metals Society of China, 16(1), 110-116 (2006).

Liu, X., y Adams, J. B., Grain-boundary segregation in Al-10\% Mg alloy at hot working temperatures. Acta mater, 3467-3476 (1998).

Ma, T., y den Ouden, G., Softening behaviour of Al-Zn-Mg alloys due to welding. Materials Science and Engineering: A, 266(1-2), 198-204 (1999).

Maury R., H., Niebles, E. E., y Torres, J., Diseño para la fabricación y ensamble de productos soldados: Un enfoque metodológico y tecnológico. Barranquilla: Uninorte (2009).

Montgomery, D., Diseño y análisis de experimentos. México: LIMUSA WILEY (2008).

Niebles, E. E., y Arnedo, W. G., Procedimientos de Soldadura y Calificación de Soldadores: Una propuesta de enseñanza y guía de aplicación para la Industria. Información Tecnológica, 20(3), 19-30 (2009). 
Oguocha, L. N., Adigun, O. J., y Yannacopoulus, S., Effect of sensitization heat treatment on properties of Al-Mg alloy AA5083-H116. Journal of Materials Science, 4208-4214 (2008).

Otto, K., y Wood, K. L., Product Design. Techniques in reverse engineering and new product development. New jersey: Prentice Hall (2001).

Padilha, A., y Siciliano Jr., F., Encruamento, recristalizaçao, crescimento de grao e textura. Sao Paulo: ABMAsociaçao Brasilera de Metalurgia e Materiais (2005).

Park, J., y Ardell, A., Correlations between microestructure and calorimetric behavior of aluminum alloy 7075 and Al.Zn-Mg alloys in various tempers. Material Science and Engineering: A, 114, 197-203 (1989).

Pfeifer, T., y Gawrysiuk, W., Technology for automated MIG-welding of high-strength aluminium alloys and the properties of the welded joints. Welding International, 20(7), 509-515 (2006).

Ponce, H., La matriz foda: alternativa de diagnóstico y determinación de estrategias de intervención en diversas organizaciones. Enseñanza e Investigación en Psicología, 12(1), 113-130 (2007).

Ramírez, J. L., Procedimiento para la elaboración de un análisis FODA como una herramienta de planeación estratégica en las empresas. Ciencia Administrativa.(2), 54-61 (2009).

Rossini , N., Dassisti, M., Benyounis, K., y Olabi, A., Methods of measuring residual stresses in components. Materials and Design(35), 572-588 (2012).

Saunders, N., y Miodownik, A., CALPHAD Calculation of Phase Diagrams.A comprehensive guide. Guilford UK: PERGAMON (1998).

Scott, D., y Totten, G., Analytical Characterization of Aluminum, Steel, and Superalloys. Boca Raton: Taylor \& Francis Group. (2006).

Skillingberg, M., y Green, J., Aluminum Applications in the rail industry. Light Metal Age. (2007).

Smallman, R., y Bishop, R., Modern Physical Metallurgy and Materials Engineering. Oxford: ButterworthHeinemann (1999).

Torres, J., Evolución microestructural de la aleación de aluminio 6061 durante el proceso de soldadura MIG. Ingeniería y Desarrollo(12), 52-65 (2002).

Totten, G., y MacKenzie, D., Handbook of Aluminum Vol 2. Alloy Production and Materials Manufacturing. New York: Marcel Dekker (2003).

Ulrich, K., y Eppinger, S., Diseño y desarrollo de productos. Enfoque multidisciplinario. Mexico D.F.: Mc Graw-Hill (2004).

Unfried S., J., Garzón, C., y Giraldo, J., Numerical and experimental analysis of microstructure evolution during arc welding in armor plate steels. Journal of Materials Processing Technology, 209(4), 1688-1700. (2009).

Valderrama, J. O., Principales Aspectos sobre la Preparación de un Artículo para ser Publicado en una Revista Internacional de Corriente Principal. Información Tecnológica, 3-14. (2005).

Yazdipour, A., Shafiei M., A., y Jamshidi Aval, H., An investigation of the microstructures and properties of metal inert gas and friction stir welds in aluminum alloy 5083. SADHANA, 36(4), 505-514. (2011)

Zander, J., y Sandström, R. Modelling technological properties of commercial wrought aluminium alloys. Material \& Design, 3752-3759 (2009). 